\title{
User-Assisted Feature Correspondence Matching
}

\author{
Dan Ring, Anil Kokaram \\ dan@unworkable.org, anil.kokaram@tcd.ie \\ Sigmedia Group, School of Electronic \& Electrical Engineering, Trinity College Dublin.
}

\begin{abstract}
Feature matching is a vital stage in many image processing applications. Finding accurate correspondences is made difficult by phenomena such as occlusions, non-rigid deformations, motion blur and more. We posit that some scenarios do not have enough information for an accurate automatic solution. Although many applications are required to be automatic, there are others that can benefit from being semi-automatic, allowing the user to provide assistance to areas where the system is failing. Good examples of this exist in the media post-production world, such as multi-view scene reconstruction, sparse-to-dense disparity estimation from view matching, image mosaic'ing (digital panoramas), or even motion estimation. The presented paper describes how to incorporate user-assistance into a Bayesian feature matching framework. By adding user information in the form of intuitive Bezier curves, difficult regions can be matched with the same accuracy as easier to match areas. The presented system uses a simple optimisation scheme, giving the user real-time interactive control over the corrected matches.
\end{abstract}

Keywords: Feature-Points, Feature Matching, User-Assisted, MRF, ICM

\section{Introduction}

Feature matching is an important part of many image processing tasks, such as image registration, object tracking, multi-view scene reconstruction and depth estimation from stereo image pairs. The accuracy of these higher level tasks depends on the accuracy of matching features. In real world images, feature matching is made difficult by non-rigid object motion, blurring and poor textural content (pathological content). There is often not enough information to automatically reject incorrect matches or propose better alternative matches. Given that user intervention is typical in post-production, we propose an interactive system to allow the user to encourage better feature matches in difficult regions, examples of which are shown in Figures $2 \& 1$.

Given sets of sparse features in two frames (such as HarrisLaplace corners [6, 5] or SIFT features [8]), previous work concentrates on finding correct correspondences between them. Most matching strategies compare local "descriptors", around the sites of the features. A low descriptor distance between a pair of features indicates a likely match. Simple heuristics

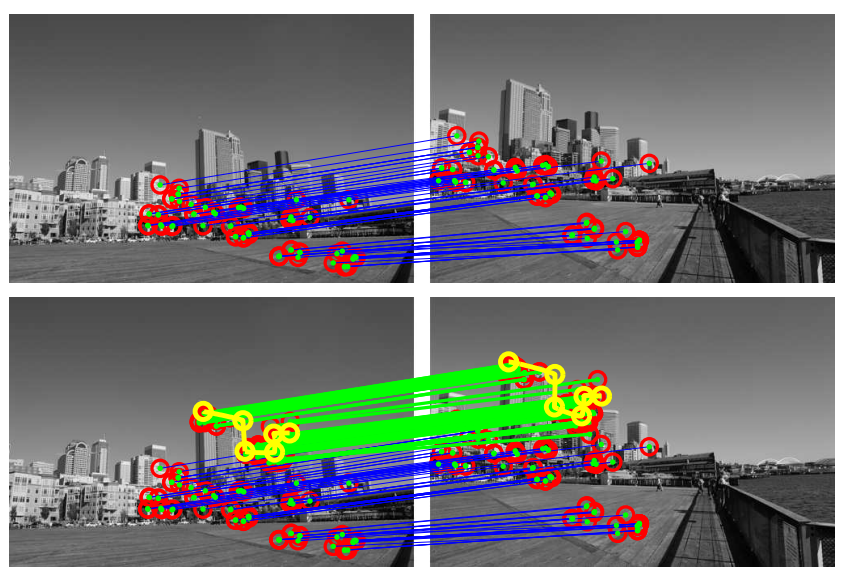

Figure 2: Example of a registration task. Top: A transformation is estimated between the two images using RANSAC, and the best correspondences are shown (blue). The model did not capture the perspective distortion and missed possible matches on a number of buildings. Bottom: two simple curves are provided by the user as a rough guide (yellow), and the previously overlooked matches are recovered (green).

such as "nearest-neighbour" and "ratio-testing" [8] attempt to match based solely on descriptor distances, disregarding the spatial context of the features. It is sensible that incorporating spatial feature context in some way, can improve feature matching performance. For example, by estimating piece-wise transformations of subsets of features [13], or requiring that at least $N$ similar feature correspondences lie in the vicinity of a potential match [11].

Recently, stereo imaging and multi-view object reconstruction are becoming more popular in post-production scenarios. Many of these applications greatly depend on the accuracy of feature correspondences. When the feature matching stage fails, digital artists paint over the problematic areas introduced by incorrect feature correspondences, such as touching up missing or erroneous depth information, or manipulating the 3D points of the reconstructed object. The presented paper uses an interactive method of correcting mistakes at the feature matching stage in order to reduce the amount of low-level correction needed by the artist. This sort of semi-automatic systems have already shown that results can be dramatically improved with minimal user intervention, the prime example 

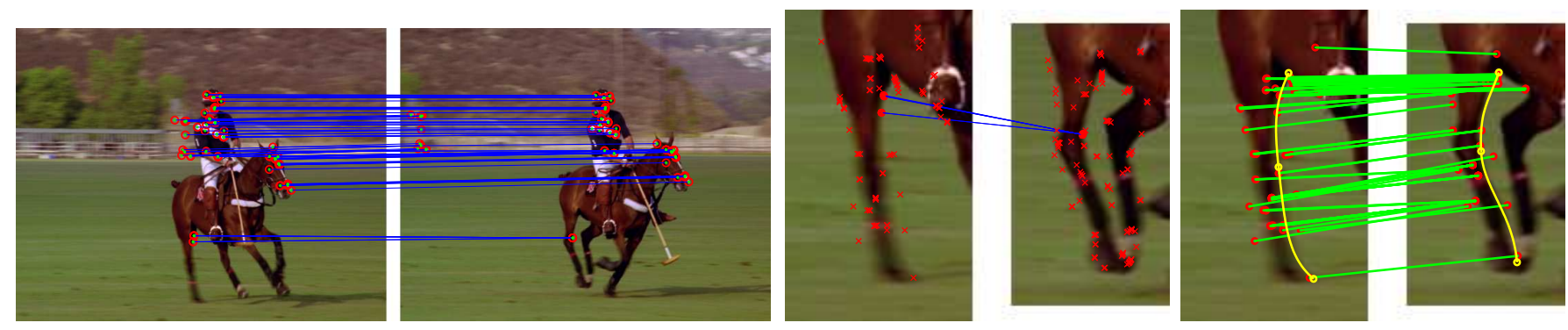

Figure 1: Some scenes do not have enough information to provide accurate feature matches. A selection of the strongest correspondences is given between the two frames in (left, blue), using the default matching scheme described in Section 3. Regardless of parameters, correspondences are never identified between the flat untextured regions of the horse, although features do indeed exist for these areas (centre, red). Following the manual addition of 2 Bezier curves to the shape of the horse's leg in the right image (yellow), matches are encouraged and identified in the difficult regions (green).

is the field of object segmentation[4, 1].

Torresani et al. [14] pose the problem of matching using an energy that combines spatial information with descriptor matching. Graph matching techniques are then used to solve the resulting optimisation problem. Despite improved matching performance, problematic regions (with pathological content) remain difficult. Figure 1 shows an example of feature correspondences similar to those found by Torresani et al. between a pair of images in a sequence. There are no feature matches found on the horse's legs due to a combination of heavy deformation and blur. However, visually it can be seen that matches should exist in these regions. Other related work that explicitly detects non-rigid deformation [10], or similarly performs quasi-dense matching [7], relies on first finding a small set of reliable matches. Again, the number of features matched in Figure 1 (centre pair) is insufficient as bootstrap matches. This reaffirms our premise that some situations simply do not have enough information to provide accurate feature correspondences.

The novelty in this paper is the development of a feature matching energy function that incorporates user information. The user is encouraged to draw two smooth curves roughly covering corresponding, yet unmatched regions. Feature matches are then encouraged in the vicinity of these curves. The advantage of this technique is that the algorithm is not restricted to the exact form of the curves, giving a good interface for user input. This provides an interactive method of correcting mistakes at the feature matching level. The new matching framework is presented next, and the process of user interaction is described. Results are discussed in Section 3.

\section{User-Assisted Matching Framework}

Following [14], consider that $P^{\prime}$ and $P^{\prime \prime}$ are the features detected in two images, and $A \subseteq P^{\prime} \times P^{\prime \prime}$ denotes the set of possible assignments between these features. A matching configuration is defined by the binary-valued vector $\mathrm{x} \in$ $\{0,1\}^{A}$. A potential correspondence $a \in A$ indexes an entry $x_{a}$ in the vector $\mathbf{x}$. The correspondence $a$ exists if $x_{a}=1$, and does not exist if $x_{a}=0$. Given a feature $p \in P^{\prime}, A(p)$ is the set of correspondences in $P^{\prime \prime}$ involving $p$. The objective is to find a matching configuration $\mathbf{x}$ that minimises an energy function as follows.

$$
E(\mathbf{x})=\lambda^{a p p} E^{a p p}(\mathbf{x})+\lambda^{u s r} E^{u s r}(\mathbf{x})+\lambda^{g e o} E^{g e o}(\mathbf{x})
$$

The components of $E(\mathbf{x})$ are the feature appearance energy, $E^{a p p}(\mathbf{x})$, our proposed user-assisted energy, $E^{u s r}(\mathbf{x})$, and the spatial consistency energy, $E^{g e o}(\mathbf{x})$, each of which is described below. The scalars $\lambda^{a p p}, \lambda^{u s r}$ and $\lambda^{g e o}$ weight the contribution of the respective energy terms.

Feature Appearance, $E^{a p p}(\mathbf{x})$ : The function $E^{a p p}(\mathbf{x})$ measures the dissimilarity between a pair of features by comparing the pixel neighbourhoods around the feature sites. The image regions around the sites are described by SIFT descriptors [8] to allow for high amounts of geometric variation, such as non-rigid deformations. $E^{a p p}(\mathbf{x})$ is therefore given by:

$$
E^{a p p}(\mathbf{x})=\sum_{a \in A(p)} x_{a}\left\|d\left(I^{\prime}, \mathbf{p}\right)-d\left(I^{\prime \prime}, \mathbf{q}\right)\right\|
$$

where $d\left(I^{\prime}, \mathbf{p}\right)$ and $d\left(I^{\prime \prime}, \mathbf{q}\right)$ are the SIFT descriptors calculated about sites $\mathbf{p}$ and $\mathbf{q}$ of features $p$ and $q \in A(p)$.

User Assistance, $E^{u s r}(\mathbf{x})$ : In this energy, cubic userdefined Bezier curves encourage correspondences in difficult to match regions. Bezier curves are already used in most image and video editing and compositing tools, making them a natural choice for user input in this situation. Imagine a region corresponding between two images exists, but is unable to be matched. In this case, the user marks the region in both images with a rough Bezier curve, an example is shown in Figure 1 (right). The cubic Bezier curve equation parameterised by $t \in\{0,1\}$ is given as follows:

$$
\mathbf{B}(t)=(1-t)^{3} \mathbf{P}_{\mathbf{0}}+3(1-t)^{2} t \mathbf{P}_{\mathbf{1}}+3(1-t) t^{2} \mathbf{P}_{\mathbf{2}}+t^{3} \mathbf{P}_{\mathbf{3}}
$$

where $\mathbf{P}_{\mathbf{0}}, \mathbf{P}_{\mathbf{1}}, \mathbf{P}_{\mathbf{2}}$, and $\mathbf{P}_{\mathbf{3}}$ are $2 \mathrm{D}$ control points in the image defining the shape of the curve. 
Intuitively, we want to use the curves as a transform function, taking locations of features around the curve in image one, and projecting them onto corresponding locations about the second curve in image two. It is not expected that the projected feature locations will match exactly to features in the second image, but the idea is to use the transformation as a soft constraint to encourage matches in the vicinity of the projected locations.

Imagine two curves $\mathbf{B}_{\mathbf{1}}(t)$ and $\mathbf{B}_{\mathbf{2}}(t)$ belonging to images one and two respectively. For a location $\mathbf{p}$, the value of the curve parameter $t$ giving the lowest distance between $\mathbf{p}$ and the curve $\mathbf{B}_{\mathbf{1}}(t)$ is found, and used to calculate the corresponding location on the second curve, $\mathbf{B}_{\mathbf{2}}(t)$. Using the local gradients of the curves, the vector angle and distances between the original and projected points are preserved. This curve transform function $\mathbf{f}$ is defined as follows:

$$
\begin{gathered}
\mathbf{f}(\mathbf{p})=\mathbf{B}_{\mathbf{2}}\left(n\left(\mathbf{B}_{\mathbf{1}}, \mathbf{p}\right)\right)+\rho\left[\begin{array}{cc}
\cos (\theta) & \sin (\theta) \\
-\sin (\theta) & \cos (\theta)
\end{array}\right] \\
n(\mathbf{B}, \mathbf{p})=\underset{t}{\arg \min }\|\mathbf{B}(t)-\mathbf{p}\|
\end{gathered}
$$

where:

$$
\begin{gathered}
\rho=\left\|\mathbf{B}_{\mathbf{1}}\left(n\left(\mathbf{B}_{\mathbf{1}}, \mathbf{p}\right)\right)-\mathbf{p}\right\| \\
\theta=\angle\left\{\left(\mathbf{p}-\mathbf{B}_{\mathbf{1}}\left(n\left(\mathbf{B}_{\mathbf{1}}, \mathbf{p}\right)\right)-\left.\frac{d \mathbf{B}_{\mathbf{1}}(t)}{d t}\right|_{t=n\left(\mathbf{B}_{\mathbf{1}}, \mathbf{p}\right)}\right\}\right. \\
+\angle\left\{\left.\frac{d \mathbf{B}_{\mathbf{2}}(t)}{d t}\right|_{t=n\left(\mathbf{B}_{\mathbf{1}}, \mathbf{p}\right)}-\left.\frac{d \mathbf{B}_{\mathbf{1}}(t)}{d t}\right|_{t=n\left(\mathbf{B}_{\mathbf{1}}, \mathbf{p}\right)}\right\}
\end{gathered}
$$

The function $n(\mathbf{B}, \mathbf{p})$ finds the parameter $t$ of the curve closest to the location $\mathbf{p}$. The numeric solution to this can be found in Graphic Gems [9]. We now have to incorporate the function $\mathbf{f}$ into an energy function. Given two features $p$ and $q$ from images one and two respectively, we propose the function $E^{u s r}(\mathbf{x})$ that encourages matches where the projected location $f(\mathbf{p})$ and feature location $\mathbf{q}$ are low:

$$
E^{u s r}(\mathbf{x})=\sum_{a \in A(p)} x_{a} \frac{\|\mathbf{f}(\mathbf{p})-\mathbf{q}\|^{2}}{r^{2}}
$$

where $r$ is a scalar to weight the disparity between $\mathbf{f}(\mathbf{p})$ and q. To limit the influence of the curves to the difficult regions, the variable $r$ is set to some distance. The value of $r$ depends on how tightly we want to constrain the projected sites about the transformation $\mathbf{f}$, and will vary depending on the matching difficulty of the region. By limiting the transformation to a specific area, it is possible to add multiple curves, enabling the correction of multiple difficult regions in the same image.

Encouraging Spatial Smoothness, $E^{g e o}(\mathbf{x})$ : Given a match between $p$ and $q$, we expect features in the neighbourhood of $p\left(p^{s}\right)$ to match to features in the neighbourhood of $q,\left(q^{s}\right)$. The work by Berg et al. [2] from shape-matching literature describes a global geometric agreement of deformations between a set of feature matches. We adapt this idea into our energy function $E^{g e o}(\mathbf{x})$ as follows.

$$
E^{g e o}(\mathbf{x})=\sum_{(a, b) \in N} x_{a} x_{b} \eta\left(e^{\delta_{a . b}^{2} / \sigma_{l}^{2}}-1\right)+(1-\eta)\left(e^{\alpha_{a, b}^{2} / \sigma_{\alpha}^{2}}-1\right)
$$

where:

$$
\begin{gathered}
\delta_{\left(p, p^{s}\right),\left(q, q^{s}\right)}=\frac{\|\| p-q\|-\| p^{s}-q^{s} \| \mid}{\|p-q\|+\left\|p^{s}-q^{s}\right\|} \\
\alpha_{\left(p, p^{s}\right),\left(q, q^{s}\right)}=\arccos \left(\frac{p-q}{\|p-q\|} \cdot \frac{p^{s}-q^{s}}{\left\|p^{s}-q^{s}\right\|}\right)
\end{gathered}
$$

The functions $\delta_{\left(p, p^{s}\right),\left(q, q^{s}\right)}$ and $\alpha_{\left(p, p^{s}\right),\left(q, q^{s}\right)}$ measure the disagreement between the distances and directions of neighbouring translational vectors respectively, with the variable $\eta \in\{0,1\}$ used to weight importance between the two. For this paper, we keep the variables $\eta, \sigma_{l}^{2}, \sigma_{\alpha}^{2}$ fixed at $0.5,4$ and 4 respectively.

The set of neighbouring feature matches $N$ is given by:

$$
\begin{gathered}
N=\left\{\left\langle(p, q),\left(p^{s}, q^{s}\right)\right\rangle \in A \times A \mid\right. \\
\left.p \in N_{p^{s}} \vee q \in N_{q^{s}} \vee p^{s} \in N_{p} \vee q^{s} \in N_{q}\right\}
\end{gathered}
$$

For example, given a match between features $p \in P^{\prime}$ and $q \in$ $P^{\prime \prime}, p^{s} \in P^{\prime}$ is in the neighbourhood $N_{p}$ of $p$, and $q^{s} \in P^{\prime \prime}$ is in the neighbourhood $N_{q}$ of $q$. A feature $p^{s}$ is considered to be within the neighbourhood $N_{p}$ if it is within the previously defined distance $r$.

\subsection{Energy Minimisation}

Torresani et al. [14] use graph matching in order to find the optimal configuration for $\mathbf{x}$. However, the advantage of a globally optimum solution comes at the expense of relatively long processing times. To allow a more interactive experience, where the user is presented with updated results following the addition or modification of a Bezier curve, we can make some observations that allow the fast but sub-optimal ICM [3] scheme to perform well.

Firstly, we note that $E^{a p p}(\mathbf{x})$ and $E^{u s r}(\mathbf{x})$, do not depend on neighbouring feature energies, and so need only be computed once. However, $E^{g e o}(\mathbf{x})$ is dependent on neighbouring feature correspondences. The number of possible feature matches to be evaluated can be reduced by considering only those within the radius $r$ of the projection of $p, \mathbf{f}(\mathbf{p})$. This set of candidates is defined as $N_{r}(p) \subseteq A(p)$.

At the beginning of the minimisation, we pre-calculate $E^{a p p}(\hat{\mathbf{x}})$ and $E^{u s r}(\hat{\mathbf{x}})$, create a proposed match configuration $\hat{\mathbf{x}}$, and randomly assign each feature $p$ to a candidate feature in $N_{r}(p)$. At each iteration of ICM, $E^{g e o}(\hat{\mathbf{x}})$ is evaluated and used to yield $E(\hat{\mathbf{x}})$ from Equation 1 . For each feature $p, \hat{\mathbf{x}}$ is then updated to select the entry in $N_{r}(p)$ with the minimum corresponding energy in $E(\hat{\mathbf{x}})$. The algorithm terminates when there are no further changes to $\hat{\mathbf{x}}$ or at most 10 iterations have passed. The estimated configuration is given by $\hat{\mathbf{x}}$.

\section{Results \& Discussion}

Ground truth feature correspondences are very difficult to generate in real images. For our work, ground truth was generated for a set of wide baseline image pairs (45 deg. apart) using the multi-view space carving work of Starck et al. [12]. The idea is that space carving can generate very accurate, dense 


\begin{tabular}{|c|c|c|c|c|}
\hline \multirow{2}{*}{ Figure 3 } & \multicolumn{2}{|c|}{ MSE } & \multicolumn{2}{c|}{ No. of Feature Matches } \\
& Before & After & Before & After \\
\hline (top) & 69.71 & 67.82 & 136 & 199 \\
(middle) & 0.34 & 0.63 & 99 & 148 \\
(bottom) & 56.58 & 47.52 & 198 & 270 \\
\hline
\end{tabular}

Table 1: Matching results before and after user interaction.

models of $3 \mathrm{D}$ objects given a large amount of views (8 in this case), and the correspondences between pairs can then be generated from the estimated 3D meshes. The MSE between our estimated feature matches and the ground truth set then gives a measure of the match quality.

For the non-user defined matching (Method $\mathrm{N}$ ), correspondences are found by nearest-neighbour matching of SIFT descriptors of the features. Using the spatial consistency constraint of [11], a match $(p, q)$ also requires at least $k$ similar matches in the neighbourhoods $N_{p}$ and $N_{q}$, for our experiments, we set $k=3$, and the neighbourhood radius $r=15$. Matches are also rejected if the descriptor differences are above a threshold, which we set at 0.5. Good values of $\lambda^{a p p}, \lambda^{u s r}$ and $\lambda^{g e o}$ for Equation 1 were found experimentally to be $0.707,5$ and 5 respectively. Results before and after user interaction are presented in Table 1, and shown visually in Figures 3.

It is encouraging that the MSE over all the features (user and automatic) does not change much from the MSE over the auto feature matching alone. In Figures 3, (top) \& (bottom), the high degree of ambiguity in matching due to blur causes a higher MSE, while in Figure 3 (middle) the highly textured regions serve to lower the MSE. In both cases, the image conditions affect user and automatic features alike. In general then, the user generated feature matches are as good as the automatic ones, and the algorithm successfully incorporates this new information. Additional examples of user-assisted matching are shown in Figures 4, 5 and 6.

The results show that the algorithm is successful at increasing dramatically the number of useful matches with minimal intervention. Our future work explores the impact these new matches can have on the performance of various cinema postproduction applications e.g. tracking and object segmentation.

\section{References}

[1] Xue Bai and Guillermo Sapiro. A geodesic framework for fast interactive image and video segmentation and matting. In Computer Vision, 2007. ICCV 2007. IEEE 11th International Conference on, pages 1-8, 2007.

[2] Alexander C. Berg, Tamara L. Berg, and Jitendra Malik. Shape matching and object recognition using low distortion correspondences. In CVPR, pages 26-33, Washington, DC, USA, 2005. IEEE Computer Society.

[3] J. Besag. On the statistical analysis of dirty pictures.
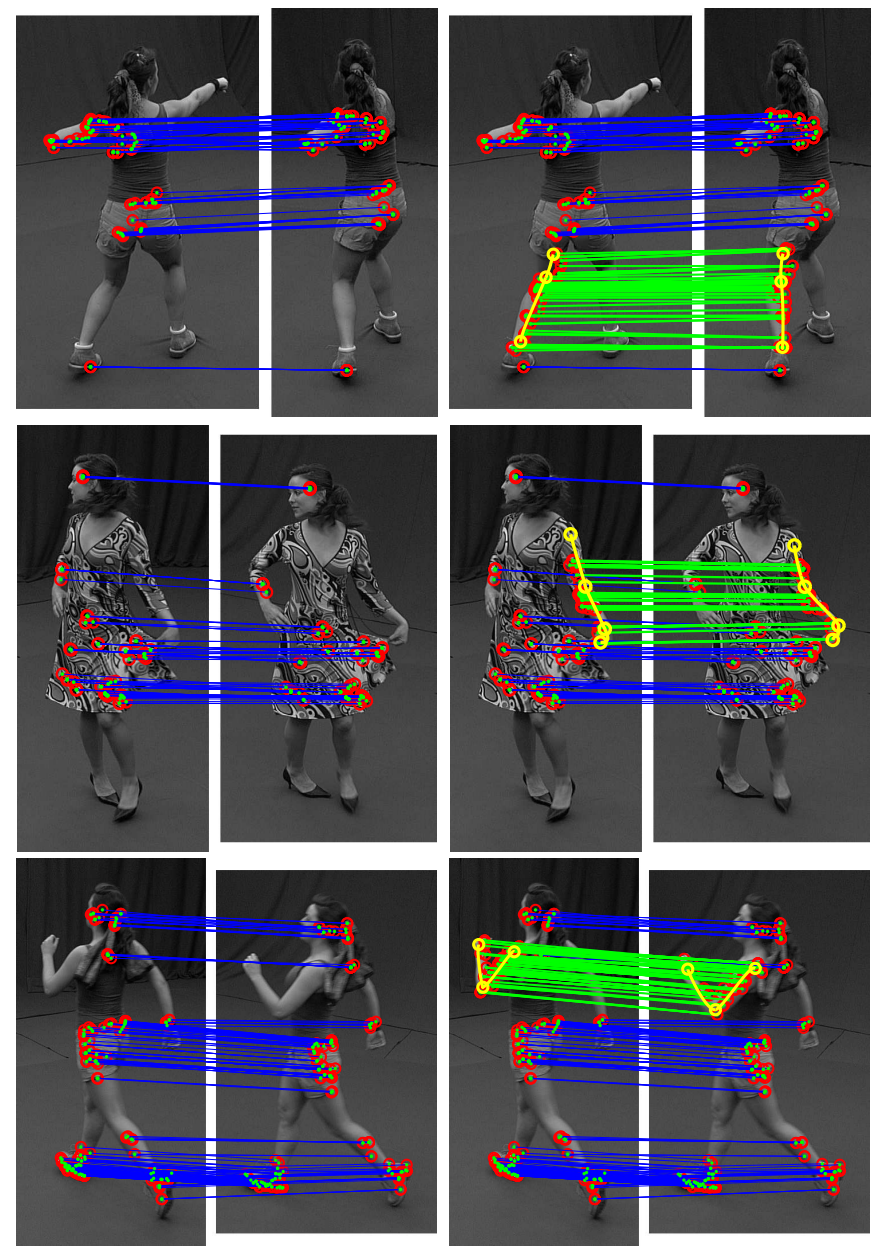

Figure 3: Scenes from a multi-view camera set-up. The pairs of images on the left show a sample of the strongest correspondences (blue) between the images using the scheme $N$. Matches are generally not found on the flat texture-less regions of the the legs are arms. In the images on the right, the user supplies corresponding Bezier curves (yellow) along the lines of limbs without matches, e.g. left leg (top-right), and left arms (middle- and bottom-right). Results of our guided matching are then shown in green on the right.

Journal of the Royal Statistical Society, Series B, 48:259302,1986 .

[4] Antonio Criminisi, Toby Sharp, and Andrew Blake. Geos: Geodesic image segmentation. In ECCV (1), pages 99112, 2008.

[5] C. Harris and M. Stephens. A combined corner and edge detector. In 4th Alvey Vision Conference, pages 147 - 151, 1988.

[6] Cordelia Schmid Krystian Mik. Bernardiolajczy. Scale \& affine invariant interest point detectors. IJCV, 60(1):6386, 2004. 


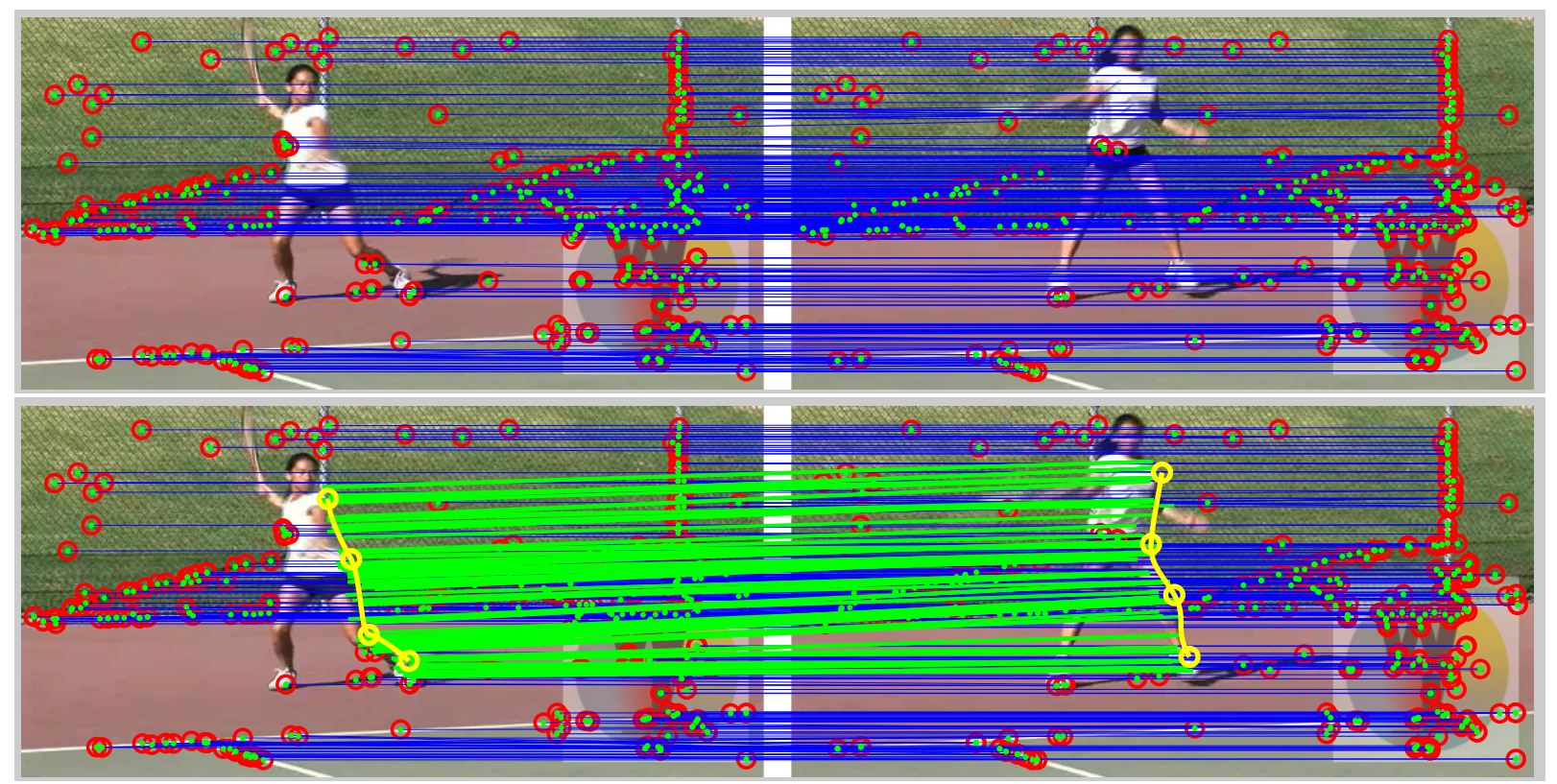

Figure 4: This example is interesting, although there are many correct matches in the scene, there are hardly any matches for the tennis player (top, blue). This is probably due to the non-rigid deformation and motion blur exhibited by the athlete. Drawing a simple set of roughly placed curves instantly generates reasonable matches for the marked side of the player. Note that the content between the frames has changed (for example, the players left leg has turned inwards), and so how useful the new features are depends largely on the target system.

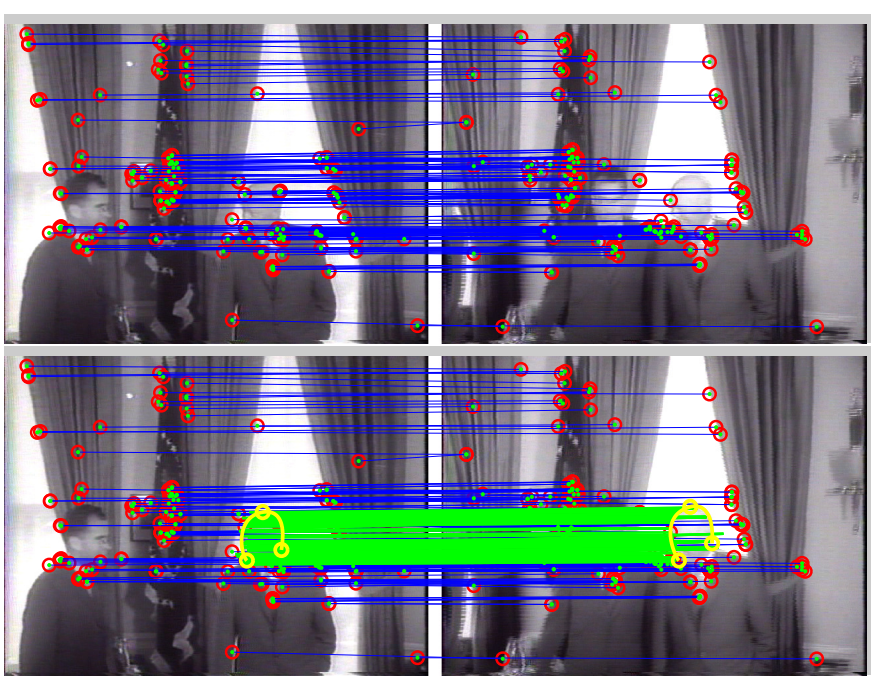

Figure 5: In this example severe interlacing artefacts make it very difficult to detect features. Notice that many of the originally matched features are actually incorrect (top, blue). After marking the head of the subject with a pair of curves, the algorithm has sufficient information to perform better matching.

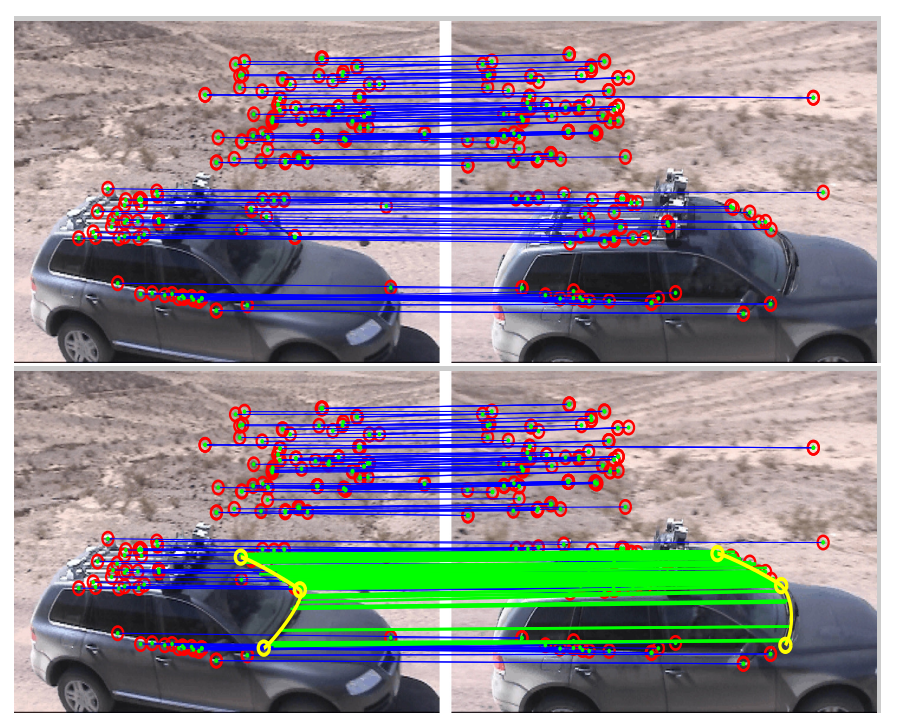

Figure 6: Feature matching is hindered in this video due to motion blur and reflection, particularly around the windscreen. Once a simple curve is drawn, the proposed system is able to identify many correct matches. As the car does not deform non-rigidly, the matching accuracy is higher than previous examples. (The remaining curves of the windscreen were intentionally omitted to give a clearer picture). 
[7] Maxime Lhuillier and Long Quan. Match propogation for image-based modeling and rendering. IEEE Trans. Pattern Anal. Mach. Intell., 24(8):1140-1146, 2002.

[8] David G. Lowe. Distinctive image features from scaleinvariant keypoints. International Journal of Computer Vision, 60(2):91-110, November 2004.

[9] Philip J. Schneider. An algorithm for automatically fitting digitized curves. pages 612-626, 1990.

[10] I. Simon and S.M. Seitz. A probabilistic model for object recognition, segmentation, and non-rigid correspondence. In Computer Vision and Pattern Recognition, 2007. CVPR '07. IEEE Conference on, pages 1-7, June 2007.

[11] J. Sivic and A. Zisserman. Video Google: Efficient visual search of videos. In J. Ponce, M. Hebert, C. Schmid, and A. Zisserman, editors, Toward Category-Level Object Recognition, volume 4170 of LNCS, pages 127-144. Springer, 2006.

[12] J. Starck and A. Hilton. Surface capture for performance based animation. IEEE Computer Graphics and Applications, 27:21-31, 2007.

[13] P. H. S. Torr. Geometric motion segmentation and model selection. Phil. Trans. Royal Society of London A, 356:1321-1340, 1998.

[14] Lorenzo Torresani, Vladimir Kolmogorov, and Carsten Rother. Feature correspondence via graph matching: Models and global optimization. In ECCV (2), pages 596-609, 2008. 